\title{
Un análisis comparativo entre algunas publicaciones especializadas en educación en América Latina
} A comparative analysis among some specialized
publications on education in Latin America

Sofía Botero Lozano, Nicolás Chaves Castillo, Lina Contreras López, Felipe Rojas Calderón, Juan Rubiano Sanabria, Sergio Salas Gómez y Diego Sánchez Parra"
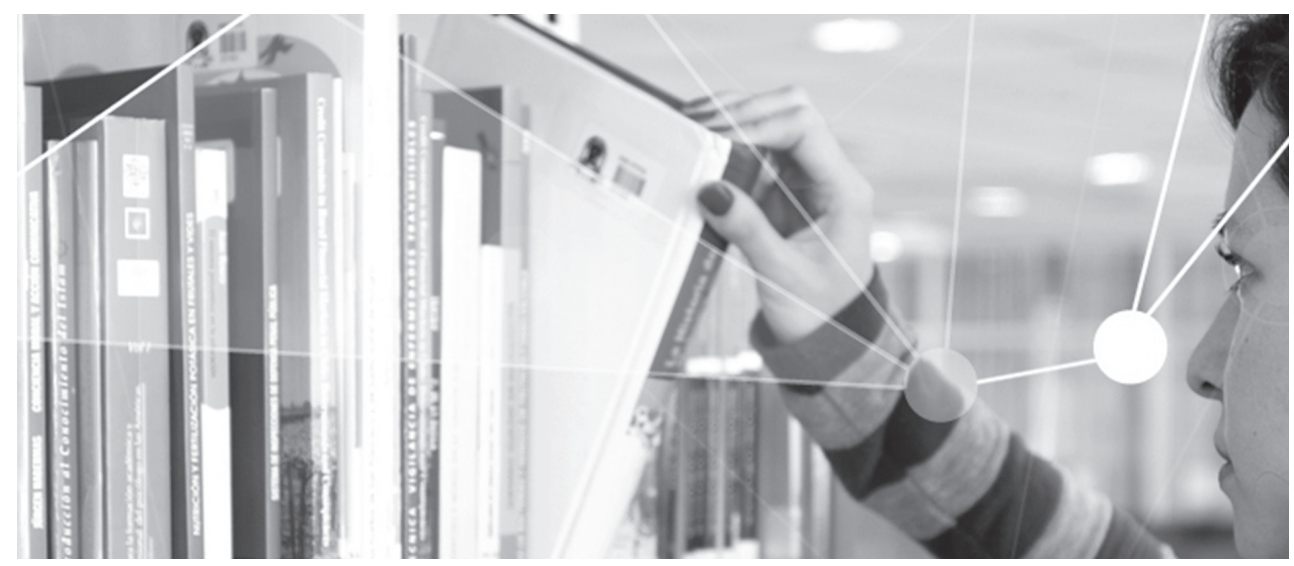

\section{Resumen}

La educación es el fundamento para la construcción de todo individuo en cada sociedad. Es con base en ella que se evalúa el desarrollo de un país, incluso cuando no sea un acto consciente. Esta investigación se basa en un estudio de carácter exploratorio donde se comparan las temáticas tratadas en publicaciones de revistas investigativas colombianas con otras de Latinoamérica, para analizar cuál es su impacto sobre los problemas sociales existentes en cada país.

Palabras clave: educación, Latinoamérica, Colombia, sistemas educativos, problemáticas sociales.

Citar este artículo como: Botero, S., Chaves, N., Contreras, L., Rojas, F., Rubiano, J., Salas, S. y Sánchez, D. (2016). Un análisis comparativo entre algunas publicaciones especializadas en educación en América Latina. Revista Papeles, 8(15), pp. 81-92.

Fecha de recibido: marzo 15 de 2016.

Fecha de aceptación: mayo 30 de 2016.

* Estudiantes del curso de Metodología de la investigación I de la Universidad Nacional de Colombia, sede Bogotá, durante el periodo 2016-I (dirigidos por el profesor Enrique Ferrer-Corredor). Correos electrónicos: Sofía Botero Lozano (lsboterol@unal.edu.co), Nicolás Chaves Castillo (nichavezca@unal.edu.co), Lina Contreras López (lmcontreraslo@unal.edu.co), Felipe Rojas Calderón (lufrojasca@unal.edu.co), Juan Rubiano Sanabria (jprubianos@unal.edu.co), Sergio Salas Gómez (ssalasg@unal.edu.co), Diego Sánchez Parra (diasanchezpa@unal.edu.co). 


\begin{abstract}
Education is fundamental for the construction of each individual part of a society in every country. The development of a country is evaluated based on its level of education, even if it is not a conscious act. This paper is an exploratory study where the topics of Colombian investigative publications are compared to those of other Latin American journals in order to judge what kind of impact they have on existing social issues in each country.
\end{abstract}

Keywords: education, Latin America, Colombia, education systems, social issues.

\title{
Introducción
}

En Colombia, y en América Latina en general, existe un amplio espectro de problemas que impactan la sociedad de manera negativa, y a un número significativo de estos se les atribuye un origen socioeconómico. Este es el caso de problemas como la desigualdad, la violencia, la corrupción y demás situaciones que pueden encontrarse en las noticias casi a diario. Estas situaciones demuestran que es menester encontrar una forma de contrarrestar los problemas que parecen crecer de manera exponencial. Tradicionalmente, la mejor solución que se puede aplicar en escenarios como el actual es la implementación de un sistema educativo que permita la inclusión de todas las comunidades, que promueva el desarrollo de temas específicos e intereses para cada región y que permita cortar los problemas de raíz, inculcando una conducta ciudadana en los jóvenes desde sus primeros años de escolaridad.

Por tal razón se investiga para encontrar soluciones puntuales a las distintas gamas de problemas anteriormente mencionados. Así, la inversión financiera con fines educativos se hace vital, y es un camino coherente que se esperaría que tomara un gobierno enfocado a la construcción de una sociedad equitativa y de calidad en cuanto a cobertura, pertinencia de los temas impartidos, pluralidad en la metodología y desarrollos temáticos específicos para cada comunidad.

Como toda inversión realizada, es de esperarse alguna rentabilidad, no solo en términos económicos de educación sino también de resultados que justifiquen dicha inversión. Sin embargo, tal como señala el Ministerio de Educación Nacional (2012) en los resultados obtenidos en las pruebas PISA 2012 sirven como un indicador de que la calidad educativa de América Latina, Colombia incluida, "Es baja en comparación con otras regiones del mundo como África central o Asia septentrional" (SCImago Jr., 2016) que, aunque se encuentran en escenarios similares, logran sortear sus dificultades para llegar a la obtención de mejores resultados en pruebas similares, lo cual es útil para determinar si las reformas realizadas están surtiendo efecto en el estudiantado e identificar las falencias para corregir los defectos.

Por lo tanto, en este trabajo se realizará un análisis comparativo entre algunas publicaciones de carácter investigativo de cuatro revistas de educación colombianas con sus pares de Brasil, Chile y México. La relación entre las investigaciones hechas en materia de educación y las problemáticas mencionadas, ambas tratadas en los artículos analizados, deberían ser presentadas en una parte significativa en los estudios realizados en las facultades de educación de distintos países de América Latina. Por lo que este documento pretende realizar un estado del arte sobre cuáles son las temáticas con mayor cantidad de estudio e investigación a nivel latinoamericano, tomando como muestra las publicaciones de los cuatro países ya mencionados, para averiguar si estas guardan relación con la búsqueda de soluciones a las problemáticas anteriormente planteadas. 


\section{Objetivos}

\section{Objetivo general}

El siguiente estudio busca revisar publicaciones de investigación en educación de países latinoamericanos. Se han tomado algunas categorías como el país origen de la publicación y del autor, el nivel académico del autor, el idioma en que están escritos los artículos y sus resúmenes $y$, finalmente, la temática tratada en cada uno de ellos. Estos criterios fueron establecidos con tal de descubrir qué tanto son tomados en cuenta los problemas de Latinoamérica a la hora de publicar un artículo con relación a la educación a través de una comparación entre estas con las publicaciones de Colombia.

\section{Marco teórico}

Antes de tener el primer acercamiento con los artículos, se realizó un estado del arte de la situación de la educación en América Latina, teniendo en cuenta a la Universidad de Sao Paulo para Brasil y su revista Educação e Sociedade, la Universidad Austral de Chile con su publicación Estudios Pedagógicos y en México al Consejo Mexicano de Investigación Educativa (COMIE) con su publicación reflejada en la Revista Mexicana de Educación; identificando algunos aspectos claves, tales como las diferencias de las políticas educativas entre la educación pública y privada, la metodología, criterios de selección de los institutos reguladores de la ciencia en los países (como Colciencias en Colombia, junto con su

\section{Objetivo específico}

Realizar la lectura de 180 resúmenes de artículos provenientes de revistas de investigación enfocadas en la educación, con 90 de ellos pertenecientes a publicaciones nacionales y 90 tomados de revistas provenientes de Brasil, Chile y México, con el fin de compararlos mediante categorías a través de una matriz, y así poder observar la incidencia de los problemas Latinoamericanos a nivel de educación y sus posibles soluciones dentro de las revistas que encabezan la investigación en nuestro continente, según el escalafón de SCImago.

\section{Metodología}

Para desarrollar el trabajo se tomaron cuatro revistas de educación colombiana, debido a que se destacan por ser publicaciones de instituciones reconocidas debido a sus investigaciones de alta rigurosidad. Fueron seleccionadas Magis, de la Pontificia Universidad Javeriana; Educación y Educadores perteneciente a la Universidad de la Sabana; conflicto con la Universidad de los Andes) y el manejo del presupuesto público destinado a la investigación.

Posteriormente, se buscó una manera de relacionar las problemáticas latinoamericanas con su aparición en las revistas de investigación, esperando encontrar que uno de los temas sobre los que se escribe en el continente fuera el de encontrar una forma de solucionar los problemas mencionados con anterioridad. Con base a esto fue que se decidió incluir en el análisis de los artículos los temas que se tratan en cada investigación, además de realizar las demás comparaciones de forma y estructura que se explicarán a continuación.
Pedagogía y Saberes (Universidad Pedagógica Nacional de Colombia, 2015) y por último la revista Voces y Saberes: Revista Latinoamericana de Educación (Universidad de los Andes, 2013). Se han escogido las anteriores revistas, ya que provienen de instituciones reconocidas por sus buenos trabajos de investigación en cuanto a educación en el contexto académico 
colombiano. Para las revistas latinoamericanas se tomaron tres revistas de tres países distintos: la Revista Mexicana de Investigación Educativa en México, la revista Estudios Pedagógicos en Chile y finalmente la revista Educaçao e Sociedade en Brasil. Estas últimas fueron escogidas por el escalafón en su respectivo país, según la clasificación de SCImago que será expuesta con mayor profundidad en los apartados correspondientes al país (SCImago Jr., 2016).

Para cada una de las revistas mencionadas se aplicaron tres criterios centrales de categorización, que son los autores, el idioma y el tema central de la investigación. Las categorías se eligieron con el fin de comparar los aspectos esenciales de cada publicación con las otras, tanto a nivel nacional como internacional, y se encuentran expresados en la tabla 1.

La primera gran categoría de clasificación es la de Autores y la segunda es la de Idioma, las cuales se tomaron como criterios de comparación para determinar cuál es el formato o el perfil de los artículos publicados en cada país para luego establecer cuál podría ser la intención de cada revista al tener ciertos estándares.
La temática de los artículos es la última categoría y es de gran importancia para este trabajo. Los siete criterios que se pueden ver en la tabla se tomaron como referentes después de haber analizado todos los resúmenes y haber extraído su tema general, los cuales se agruparon por similitud. La subcategoría Otros existe porque no fue posible agrupar algunos temas en los grupos ya existentes.

Los datos fueron recopilados en esta matriz, en la que se identificaron las características que cumplía cada artículo. Con esto se logró tener el material necesario para realizar las gráficas que permitieron la comparación de los artículos, y algunas de ellas están incluidas a lo largo del artículo.

Lo anterior permitió realizar el análisis detallado para cada país, registrados en los primeros cuatro apartados de este trabajo, que reflejan las problemáticas de cada uno, enfocado a las diferentes perspectivas educativas. En el último apartado se cumplirá el objetivo general de la investigación, al comparar los resultados unificados de los países latinoamericanos analizados con los del contexto colombiano.

Tabla 1. Categorías aplicadas a las revistas

\begin{tabular}{|c|c|}
\hline 1. Autores & \\
\hline Categoría & Subcategoría \\
\hline \multirow{6}{*}{ Número de autores } & 1.1.1 Uno \\
\hline & 1.1.2 Dos \\
\hline & 1.1.3 Tres \\
\hline & 1.1.4 Cuatro \\
\hline & 1.1.5 Cinco \\
\hline & 1.1.6 Más de cinco \\
\hline \multirow{6}{*}{ Nivel académico } & 1.2.1 Pregrado \\
\hline & 1.2.2 Especialización \\
\hline & 1.2.3 Maestría \\
\hline & 1.2.4 Doctorado \\
\hline & 1.2.5 Posdoctorado \\
\hline & 1.2.6 No especificado \\
\hline \multirow{5}{*}{ Procedencia } & 1.3.1 País de publicación \\
\hline & 1.3.2 Latinoamérica \\
\hline & 1.3.3 Norte América \\
\hline & 1.3.4 Europa \\
\hline & 1.3.5 Otros \\
\hline
\end{tabular}

Fuente: elaboración propia

\begin{tabular}{ll}
\hline 2. Idioma & Subcategoría \\
\hline Categoría & $\frac{2.1 .1 \text { Español }}{2.1 .2 \text { Inglés }}$ \\
\hline \multirow{4}{*}{ Abstracts } & $\frac{2.1 .3 \text { Francés }}{2.1 .4 \text { Portugués }}$ \\
\hline \multirow{4}{*}{ Texto } & $\frac{2.2 .1 \text { Español }}{2.2 .2 \text { Inglés }}$ \\
\hline & 2.2.3 Francés \\
\hline
\end{tabular}

\begin{tabular}{l|}
\hline 3.Tema \\
\hline 3.1 Tecnología y educación \\
\hline 3.2 Formación lectora \\
\hline 3.3 Metodología educativa \\
\hline 3.4 Políticas educativas \\
\hline 3.5 Labor docente \\
\hline 3.6 Formación docente \\
\hline 3.7 Otros \\
\hline
\end{tabular}




\section{Análisis de los resúmenes por países}

\section{Análisis en el contexto educativo colombiano}

La educación en Colombia ha sido históricamente un conjunto de contrastes culturales, políticos, estrategias erradas, ideas innovadores e inconvenientes de gran magnitud que involucran una amplia búsqueda de soluciones y definición de problemas.

Tal como se ha visto a lo largo de los seis últimos números de Voces y Silencios: Revista Latinoamericana de Educación de la Universidad de los Andes, presentar la educación en Latinoamérica y más específicamente en Colombia:

Ha llevado a muchos investigadores a buscar las inconsistencias del sistema, en cuanto a cobertura, temáticas impartidas y la protección ante amenazas en contra del ambiente educativo, lo que lleva a la promoción de estrategias que contribuyan a que este pueda mejorarse en todos los ámbitos posibles (Universidad de los Andes, 2013).

Las críticas a una educación monopolizada y conquistada por intereses económicos, desconocimiento de la cultura de algunas comunidades que habitan en la misma nación y el encasillamiento en temas que ignoran el pensamiento crítico o el pleno desarrollo cultural y humano, son algunos de los temas más representativos (catalogados en la características de políticas educativas) debido a que abarcan conceptos con el objetivo de buscar la reacción del público ante una realidad más creciente y desafiante.

La inmersión de la tecnología en un nuevo ambiente educativo, es otra de las diversas posturas de los autores colombianos Yamith Fandiño, Aurora Cardona y Jairo Galindo, más específicamente a través de las "wikis", "libros virtuales" y "ambientes de aprendizaje": "Han sido unas de las muchas propuestas para fomentar el uso de estas herramientas que tanto pueden ayudar a una educación que sufre por la falta de innovación" (Universidad de los Andes, 2014).

Con esta enorme contribución tecnológica en el área de la educación y un amplio desarrollo comercial y económico mundial, además del creciente fenómeno de la globalización, aparece entonces la necesidad de aprender un nuevo idioma, que por cuestiones comerciales es el inglés, ya que el amplio dominio de la cultura anglosajona (en especial de Estados Unidos y Gran Bretaña), con sus inventos y publicaciones, ha sido de fuerte influencia en el hombre. Este ha sido un duro golpe para algunos gobiernos que no manejaban dicho idioma, en algunos detalles, tales como el reajuste de matrices curriculares, el gran gasto de recursos en la adquisición del material indicado y sobretodo designar personal debidamente calificado para impartir la asignatura; trayendo consigo enormes dificultades, ya que las metodologías usadas en el ambiente de aprendizaje no son idóneas para la correcta implementación de tal lengua en el país.

Uno de los grandes debates en las publicaciones de educación es la inclusión de estudiantes de las regiones apartadas de Colombia; temas como la cobertura, la temática impartida y el respeto por las costumbres de cada región específica, son de gran relevancia para acercarse a los problemas educativos latinoamericanos. Sin embargo, no son recurrentes en las publicaciones de Colombia. "Esto permite un gran conflicto a la hora de pensar en el paso correcto y seguro para realizar la ejecución de políticas coherentes que satisfagan la amplia necesidad de educación en el territorio colombiano" (Universidad de los Andes, 2015).

De los noventa artículos seleccionados, veintiocho de ellos (56\%) están redactados por autores con doctorado, lo que demuestra que la cantidad de publicaciones depende del nivel de académico del autores (Gráfica 1). 
Gráfica 1. Nivel académico de los autores. Colombia.

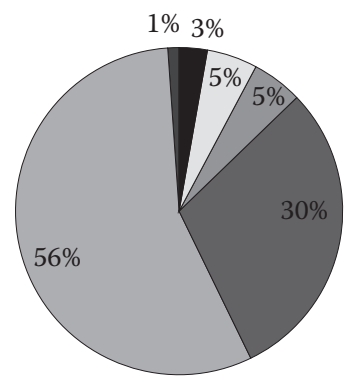

1.2 Nivel académico

$\square$ 1.2.1 Pregrado

$\square$ 1.2.2 Especialización

• 1.2.3 Maestria

$\square$ 1.2.4 Doctorado

- 1.2.5 Posdoctorado

- 1.2.6 No especificado

Fuente: elaboración propia

Gráfica 2. Procedencia de los autores. Colombia.

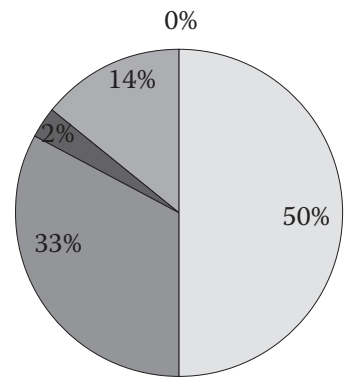

1.3 Procedencia del autor

$\square$ 1.3.1 País de publicación

$\square$ 1.3.2 Latinoamericana

- 1.3.3 Norte América

$\square$ 1.3.4 Europa

$\square$ 1.3.5 Otros

Fuente: elaboración propia

Tal como se interpreta en la Gráfica 2, la mayor cantidad de resúmenes consultados fueron realizados por autores de la misma nacionalidad (Colombia), concluyendo que no se importa talento humano en mayor cantidad, sino que se sostiene mayoritariamente el talento humano nacional.

De los noventa resúmenes analizados, la mayor parte de ellos fueron hechos en español (89 en total); sin embargo, con el fenómeno de la globalización se vuelve una necesidad publicar en diferentes idiomas, siendo predominante en inglés por ser el idioma universal, y en portugués por la influencia de Brasil en el contexto latinoamericano.

A partir de la gráfica 3 se puede obtener un análisis homogéneo en cuanto a la recurrencia en los temas de los artículos; esto permite observar la uniformidad en las concepciones de los autores, tales como la importancia de
Gráfica 3. Temas de los artículos. Colombia.

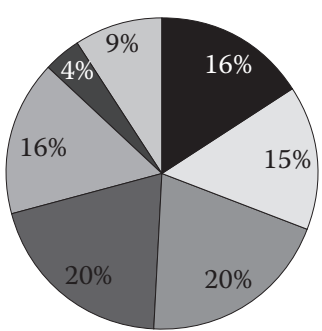

Fuente: elaboración propia
- 3.1 Tecnología y Educación

$\square 3.2$ Formación Lectora

- 3.3 Metodología Educativa

- 3.4 Políticas Educativas

$\square$ 3.5 Labor Docente

- 3.6 Formación Docente

$\square 3.7$ Otros la tecnología, la reestructuración del sistema educativo o la evaluación de los docentes al momento de abordar las problemáticas o necesidades que tiene el país en cuestiones educativas.

\section{Análisis de impacto social en la educación brasileña}

Brasil ocupa el décimo puesto a nivel mundial en SCImago en la categoría educación, y a nivel latinoamericano tiene el primer puesto. La revista que se analizó de Brasil fue Educação e Sociedade, publicada trimestralmente por el Centro de Estudios Educação e Sociedade (CEDES). Ocupa el tercer puesto a nivel latinoamericano y el 585 a nivel mundial según él Centro de Estudos Edução e Sociedade (2015).

El común denominador en la revista es la publicación por parte de académicos con títulos de doctorado y posdoctorado, teniendo los magíster un menor protagonismo, y aún menor las personas de pregrado. Esto demuestra el grado de especialización al que ha llegado la revista y explica cómo es que logra posicionarse en los puestos más altos del escalafón a nivel latinoamericano.

En esta revista todos los artículos cuentan con resúmenes en inglés y francés, mostrando lo importante que es para la revista el ser reconocidos y estudiados internacionalmente. Es de resaltar el hecho que, aunque Brasil esté en una región de habla mayormente hispánica, la 
Gráfica 4. Temas de los artículos. Brasil.

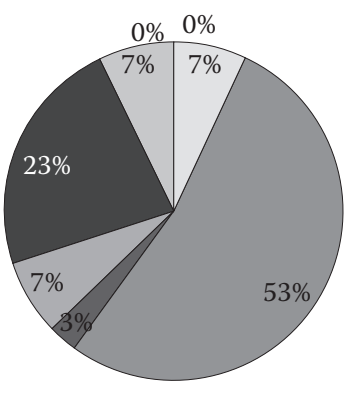

Fuente: elaboración propia
- 3.1 Tecnología y Educación

$\square 3.2$ Formación Lectora

$\square$ 3.3 Metodología Educativa

- 3.4 Políticas Educativas

$\square 3.5$ Labor Docente

- 3.6 Formación Docente

$\square 3.7$ Otros

$\square 3.8$ Educación pública vs educación privada

revista utiliza poco el idioma español, con solo dos resúmenes de artículos escritos en español, lo que podría indicar que, quizá, los intereses académicos de Brasil no están enfocados en Latinoamérica.

Dejando los resúmenes de lado, el cuerpo de los artículos está escrito únicamente en portugués, con solo tres de treinta artículos que cuentan con una traducción completa en un idioma diferente (dos en español y uno en inglés). Es notable también que la revista cuente con solo un $25 \%$ de participación extranjera, predominando así los autores portugueses.

Al hacer un análisis de los temas más frecuentes en la revista, se evidencia la gran importancia que reciben las políticas públicas, unidas casi siempre con los artículos que tratan sobre políticas educativas. También, son numerosos los artículos que tratan sobre las diferencias entre educación pública y educación privada, y lo que esto puede significar dentro de la sociedad brasilera en general, puesto que, usualmente, solo las personas de bajos recursos son quienes acceden a la educación pública. Estos dos temas se convierten en elementos muy importantes a la hora de buscar la solución de los principales problemas de educación en Latinoamérica, entre los cuales están: "Mala calidad educativa, poca inversión en este sector, entre otros" (García \& Zinny, 2014).
Los porcentajes pertenecientes a metodología educativa y labor docente suelen relacionarse entre sí a la hora de escribir los artículos, aunque siempre prevalece alguno de los dos. Están enfocados principalmente hacia la baja calidad de la educación a la que tratan de dar una explicación y posibles soluciones.

La categoría Otros tiene un gran porcentaje por la variedad de temas que se manejan, pero estos temas no tienen más de un artículo; por ejemplo, la educación para niños con discapacidad, el cual solo tiene un artículo en Brasil y a nivel global del trabajo no hay más de cinco.

\section{De un análisis con origen pedagógico aplicado a la educación de Chile}

Chile está en el puesto 36 a nivel internacional dentro del escalafón de SCImago, al mismo tiempo que es el tercero en Latinoamérica. Se tomó como revista para analizar la que ocupa el primer puesto dentro del país: Estudios Pedagógicos de la Universidad Austral de Chile. Aunque es la primera en el país, ocupa el quinto puesto en Latinoamérica y el 597 a escala mundial (SCImago Jr., 2016).

La revista cuenta con un número semestral, además de tener un número especial que se presenta cada año. Para este trabajo se tomaron como muestras las tres últimas publicaciones del año 2015, vol. 41, no.1, 2, más su número especial de dicho año; de los cuales se escogieron aleatoriamente 30 artículos para realizar el análisis.

Gráfica 5. Procedencia de los autores.

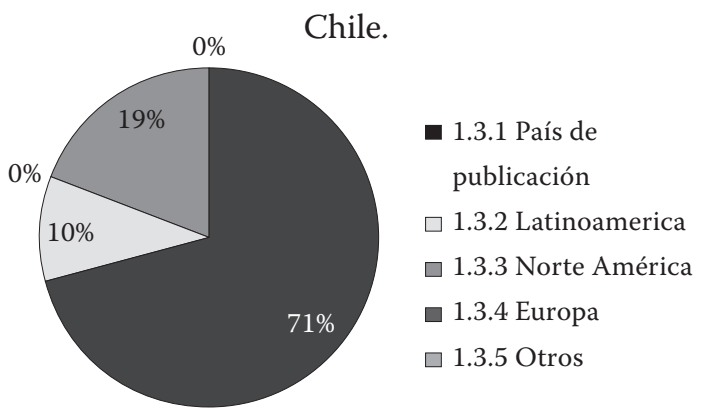

Fuente: elaboración propia 
Se encontró que en la revista Estudios Pedagógicos el $50 \%$ de los artículos publicados fueron hechos por dos autores, dándole prioridad a los autores afiliados a entidades nacionales (Gráfica 5), aunque se proyecta a una audiencia internacional, puesto que la totalidad de los resúmenes están escritos en tres idiomas: el español natal, inglés y portugués; lo que guarda coherencia con lo expuesto en capítulos anteriores sobre la relación que existe entre lenguaje e impacto en la comunidad académica mundial. No obstante, el 100\% de los artículos fue publicado en español, incluso si los autores de estos son procedentes de Europa y demás países no hispanohablantes.

En el análisis de esta publicación los datos referentes al nivel académico de los autores no se pudieron recolectar en su totalidad, ya que no aparecían en los resúmenes, y Chile no cuenta con una base de datos como CvLAC en Colombia para obtener esta información.

En la Gráfica 6 se evidencian tres grandes ejes temáticos entre los resúmenes: Tecnología y Educación, con el 17\%; Metodología Educativa, con el 27\%; y la categoría Otros, con el $23 \%$. Así, se evidencia que la mayoría de los artículos son destinados a cubrir problemas internos de la educación chilena, tales como la metodología con la que se enseña o la formación de futuros pedagogos. Por otro lado, las Políticas Educativas, que podrían enfocar los proyectos educativos institucionales hacia una visión innovadora y abierta a cambios, es cubierta por tan solo $10 \%$ de los artículos,

Gráfica 6. Temas de los artículos.

Chile.

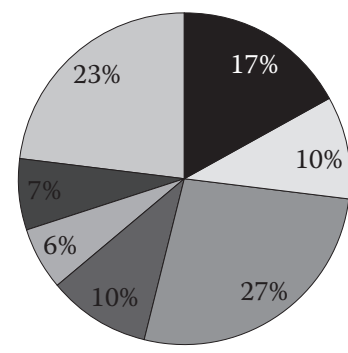

3.1 Tecnología y Educación

$\square 3.2$ Formación Lectora

$\square$ 3.3 Metodología Educativa

— 3.4 Políticas Educativas

$\square$ 3.5 Labor Docente

- 3.6 Formación Docente

$\square 3.7$ Otros

Fuente: elaboración propia además de que las temáticas relacionadas con proponer soluciones a los mayores problemas de la educación cuentan con un porcentaje aún menor a este, tanto así que por sí solos no alcanzan a tener un valor pertinente dentro de las gráfica, y son incluidos dentro de la categoría Otros. Algunos de estos artículos tratan temas como la formación de los estudiantes de educación básica para ser ciudadanos o la segregación socio-económica en el sistema educativo; temas que, pese a ser importantes, presentan una incidencia baja en los números del año 2015.

\section{Análisis investigativo para el desarrollo educativo de México}

Tomando el escalafón internacional de SCImago, el cual ubica a México en el puesto 31 a nivel internacional y a nivel latinoamericano en el segundo, se tomó para este análisis la Revista Mexicana de Investigación, que es dirigida por el Consejo Mexicano de Investigación Educativa (COMIE). Esta se posiciona en el segundo lugar en su país, siendo a la vez calificada en el sexto lugar a nivel de Latinoamérica, aunque ocupa el puesto 603 en el mundo (SCImago Jr., 2016).

Se encontró que la Revista Mexicana de Investigación Educativa tiene una distribución parcialmente equitativa sobre el número de autores que participan en la investigación de cada artículo, al estar el 50\% distribuido entre artículos trabajados por dos o tres personas; el $28 \%$ por un único autor y un número significativo de artículos resultantes del trabajo en equipo de seis personas que representa el $16 \%$. Otro dato arrojado por la tabla es que aunque el $90 \%$ de los autores es mexicano, el porcentaje restante es mayormente cubierto por académicos europeos y en menor escala por latinoamericanos; sin contar con la colaboración de norteamericanos, lo que resulta sorpresivo dada la cercanía geográfica con México y el resto de América Latina. "Además de revelar el poco o nulo interés que se tiene por 
Gráfica 7. Temas de los artículos. México.

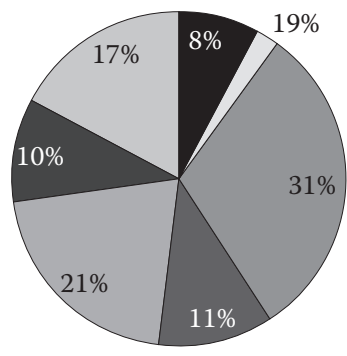

- 3.1 Tecnología y Educación

$\square 3.2$ Formación Lectora

- 3.3 Metodología Educativa

- 3.4 Políticas Educativas

$\square$ 3.5 Labor Docente

- 3.6 Formación Docente $\square 3.7$ Otros

Fuente: elaboración propia

las instituciones educativas que se encuentran dentro del continente" (Consejo Mexicano de Investigación Educativa (COMIE), 2015).

Dado que la totalidad de los artículos cuentan con resúmenes tanto en español como en inglés, se puede deducir que la revista, así no cuente con una proporción notable de autores de habla inglesa, se presenta para un público amplio, pues maneja el idioma hasta el punto de concentrar sus esfuerzos en la posibilidad de publicar en ese idioma y así tratar de llegar a tener reconocimiento internacional en este aspecto, pues no hay un solo artículo que cuente con sus resúmenes en francés, en portugués o en otro idioma.

Por último, al analizar los temas en la revista, se puede concluir que hay tres temas representativos: la Metodología educativa, la Labor docente y la categoría de Otros. Esto demuestra la importancia que representa el implementar medidas dentro de las clases que busquen maneras de innovar en materia de educación desde adentro, mediante investigaciones o trabajos de campo que arrojen resultados que puedan ser posteriormente utilizados. También se le da gran importancia a la labor del docente dentro el sistema educativo y, finalmente, se muestra que la categoría de Otros es mayor a temas como la formación de nuevos pedagogos o la formación en tecnología y educación.

En México existe un gran interés y tendencia por producir artículos acerca de la inclusión social y en educación en cuanto a la población indígena de este país; se buscan, recogen y analizan diversas cantidades de información con el objetivo de proponer soluciones a problemáticas que se identifican dentro de la población estudiada. Además, es importante resaltar que la investigación en educación se entiende en México como una investigación para las ciencias sociales, ya que sus artículos manifiestan expresamente temas relacionados con la inclusión de regiones periféricas, la pertinencia de algunos temas para comunidades específicas y la importancia de la cultura para la impartición de la educación, lo que da a conocer la relevancia de la sociedad en los procesos educativos.

Unas conclusiones sólidas en el caso de México, es que, el trabajo realizado, contribuye a cumplir los objetivos planteados por los investigadores, y puesta sobre la mesa una posible solución a problemas como la influencia de una sociedad que ha sufrido mucha violencia en los ambientes educativos, sumado con la necesidad de un sistema incluyente para todos los estudiantes.

\section{Perspectiva de la educación colombiana y la educación latinoamericana}

En la Gráfica 8 se presentan los temas encontrados en los noventa resúmenes analizados entre Brasil, Chile y México, donde se evidencia

Gráfica 8. Temas de artículos internacionales a nivel latinoamericano.
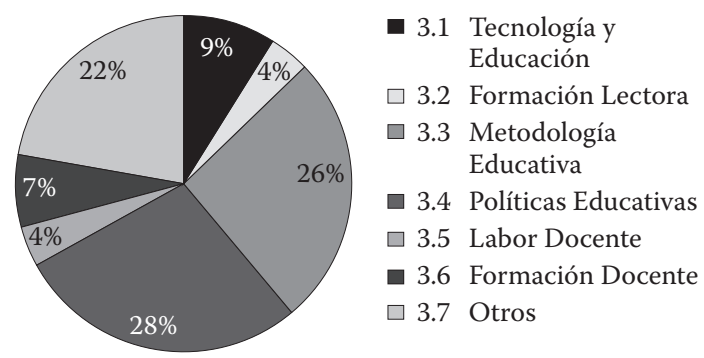

Fuente: elaboración propia 
una especie de equidad entre las temáticas que presentan los proyectos de investigación. Cabe recalcar que las necesidades y deficiencias de los sistemas educativos en los países escogidos son variables según la cultura o la postura política de actualidad.

Por otro lado, como se ve en la Gráfica 3, los temas destacados en los artículos de Colombia no presentan mayor variación de los temas latinoamericanos, mostrando que Colombia sigue el mismo patrón temático, posiblemente porque las problemáticas presentadas en el país son similares a las que se presentan a nivel general en Latinoamérica.

Se puede observar que en Colombia está más avanzada en el esfuerzo por vincular la tecnología y la educación, puesto que los artículos con este tema central representan un 16\%, mientras que en Latinoamérica solo el $9 \%$. Cifras con proporciones similares se observan en la categoría de Formación lectora, ya que el $15 \%$ de las publicaciones colombianas se centran en esta área específica del aprendizaje, mientras que en América Latina solo representan el 4\%; y en la categoría Labor docente con cifras de $16 \%$ y $4 \%$ respectivamente.

El número de artículos publicados sobre la formación docente es un poco preocupante puesto que en ambos casos representa menos del 10\%.
La categoría Otros representa solo el 9\% de las publicaciones colombianas, pero en la gráfica latinoamericana (gráfica 8) tiene una participación significativa del $22 \%$. Es aquí donde se encuentran varios artículos con temas demasiado dispersos para poder clasificarlos dentro de las categorías ya establecidas. Sin embargo, es importante destacar que se encontraron algunos que trataban temas como formación ciudadana en niños de preescolar, el ambiente escolar, la etnografía escolar y desigualdad socioeconómica dentro del sistema educativo.

Es pertinente mencionar, además, que la inclusión del sistema CvLAC como sistema para el reconocimiento de los autores de las diversas publicaciones, ha sido una gran ventaja al momento de comparar la manera en la cual se registra la obra en temas científicos (Currículum Vitae de Latinoamérica y el Caribe (CvLAC), 2016). Por el contrario, en países como Chile o Brasil fue muy difícil la búsqueda del grado de escolaridad del autor; ventaja que en Colombia se subsana con CvLAC.

La explotación y propagación de temas como los anteriores podrían influir en cambiar y solucionar las problemáticas que se han ido mencionando a lo largo de este trabajo, así también los temas más representativos en ambas gráficas, los cuales son metodología educativa y políticas educativas.

\section{Conclusiones}

Luego de analizar los 180 artículos y clasificarlos en categorías, fue posible determinar que los temas más recurrentes en Latinoamérica y Colombia fueron las políticas educativas y la metodología educativa, mientras que las temáticas formación lectora, labor y formación docente representan las menores cifras.

Tras analizar las temáticas, es válido realizar una serie de conclusiones en materia de los temas que se están discutiendo en revistas de educación, tanto a nivel nacional como internacional.
En primer lugar, es preocupante que los artículos que hacen parte de la categoría Otros, los cuales tratan de temas como la baja calidad en la educación en Latinoamérica, su falta de inclusión y las grandes brechas entre la educación pública y privada, entre otros, no se publiquen de forma representativa. Es por esto que se vuelve una necesidad el analizar y criticar las políticas públicas que no están llevando a una mejora en la educación, sino que, más bien, convierten la educación en un servicio y no en un derecho elemental. Esto se 
puede ver en programas como "Ser pilo paga" en Colombia o "ProUni" en Brasil, que ofrecen créditos a los estudiantes (que después tienen que ser pagados con intereses), en vez de invertir directamente sobre las instituciones de educación pública.

Ahora, si este es el caso de la educación en general, al analizar los artículos se pudo observar el poco interés que para estos estudios realizados evidencian en la educación para niños con discapacidad física o mental. El poco trato de este tema muestra que la educación para estos niños es de acceso muy limitado en general y seguramente de acceso nulo para personas de escasos recursos, lo que contribuye al aumento de la desigualdad entre las personas con discapacidad y las personas en plena facultad, y volviendo más difícil que las personas con discapacidad, por su bajo acceso a la educación, puedan obtener un trabajo digno y de calidad, negando entonces la posibilidad de que, en un futuro, aspiren a una mejor calidad de vida.

Uno de los temas más recurrentes en las investigaciones en Latinoamérica es la metodología educativa, en el cual se incluye la calificación al docente. Este tema va principalmente dirigido hacia la calidad de la educación, mostrando cómo este problema se ha vuelto muy relevante en el contexto latinoamericano, incluyendo a Colombia (con un 20\% de frecuencia en los artículos como se ve en la gráfica 7) con problemas como el paro de educadores en contra de que los califiquen.
Las publicaciones conforme al tema de la tecnología y la educación no tienen un porcentaje muy relevante en Latinoamérica (solo un 9\%), lo que da lugar a un atraso en este aspecto, donde se priva el desarrollo de nuevas ideas. Se debe aplicar más esfuerzo de parte de Latinoamérica hacia este enfoque, ya que muchos de los artículos de temas como políticas públicas se vuelven repetitivos en sus análisis y en sus conclusiones, dejando de producir nuevo contenido y perdiendo recursos que se podrían usar para explotar la mina de oro que se tiene con las nuevas tecnologías que se están desarrollando, y para aplicarlas en la educación.

Aunque este trabajo reconoce la gran labor que realizan los investigadores en materia de educación, tanto a nivel colombiano como a nivel mundial, es posible encontrar que, pese a que existen quienes intentan dar con una solución a los problemas más urgentes de la educación en general, las tendencias temáticas no se encuentran precisamente en este campo. Tal vez se deba a la falta de promoción de estas ideas, o tal vez sea porque existe una amplia variedad de temas de estudio dentro de los cuales es posible realizar un cambio en la sociedad. No obstante, por mucho que se pueda innovar en campos como el tecnológico, en la formación docente o en las políticas educativas de una región en particular, no se puede llegar a ser una mejor sociedad si antes no se cambia el sistema desde adentro, buscando soluciones para estos problemas, y todos los que estén por venir.

\section{Referencias}

Centro de Estudos Educação e Sociedade. (2015). Educação e Sociedade (Vol. 36). Campinas: Centro de Estudos Educação e Sociedade.

Consejo Mexicano de Investigación Educativa (COMIE). (2015). Revista Mexicana de Investigación Educativa (Vol. 20). Ciudad de México: Consejo Mexicano de Investigación Educativa (COMIE).
Currículum Vitae de Latinoamérica y el Caribe (CvLAC). (2016). CvLAC. Obtenido de CvLAC: http://scienti.colciencias.gov.co:8081/cvlac/ Login/pre_s_login.do

Farfán, Garzón, Quintana, \& Sánchez. (2014). Cuadernos de Economía: Huellas y Estadísticas. Econografos, 1-43. 
García, \& Zinny, S. (28 de Abril de 2014). Revista Semana, sección Educación. Obtenido de Semana.com: http://www.semana.com/edu cacion/articulo/los-desafios-de-la-educacionen-america-latina/384841-3

Ministerio de Educación Nacional de Colombia. (2012). Colombia en PISA 2012. Bogotá D.C.: Ministerio de Educación.

Pontificia Universidad Javeriana. (2016). Magis: Revista Internacional de Investigación Educativa (Vol. 7 y 8). Bogotá D.C.: Pontificia Universidad Javeriana.

SCImago Jr. (10 de Mayo de 2016). SCImago Journal \& Country Rank. Obtenido de SCImago Journal \& Country Rank: http://scimagojr.com/

Universidad Austral de Chile. (2015). Estudios Pedagógicos (Vol. 41). Valdivia: Universidad Austral de Chile.
Universidad de la Sabana. (2015). Educación y Educadores (Vol. 18 y 19). Bogotá D.C.: Universidad de la Sabana.

Universidad de los Andes. (2013). Voces y Silencios: Revista Latinoamericana de Educación (Vol. 4). Bogotá D.C.: Universidad de los Andes.

Universidad de los Andes. (2014). Voces y Silencios: Revista Latinoamericana de educación (Vol. 5). Bogotá D.C.

Universidad de los Andes. (2015). Voces y Silencios: Revista Latinoamericana de Educación (Vol. 6). Bogotá D.C.

Universidad Pedagógica Nacional de Colombia. (2015). Pedagogía y Saberes (Vol. 43). Bogotá D.C.: Universidad Pedagógica Nacional. 